\title{
ARCHITA: an innovative multidimensional mobile mapping system for tunnels and infrastructures
}

\author{
Federico Foria $^{1 *}$, Gabriele Avancini ${ }^{1}$, Ricardo Ferraro ${ }^{1}$, Gabriele Miceli ${ }^{1}$, and Esther \\ Peticchia $^{1}$ \\ ${ }^{1}$ ETS Srl, Via Appia Nuova 59, Roma, Italia
}

\begin{abstract}
This paper presents ARCHITA: an innovative multidimensional mobile mapping system for the survey and the diagnostic of tunnels and infrastructures. The system gives the outputs of geometry and structures conditions in an integrated environment. The paper provides a brief description of the system and the successful application to support the decision-makers and the designers. Moreover, the outputs of the system are presented throughout the paper for different tunnels.
\end{abstract}

\section{The heritage infrastructure in Italy and Europe}

The European Union brought up the need of the renovation of the "European Core Network" (e.g. TEN-T, Trans European Network-Transport) railway to the European standards. The perspective is to ensure links between the different transport modes, eliminating existing choke point. The completion of the "European Core Network" for 2030 is an important effort for Italy, especially regards to the efficiency of rail and road connections and the completion of the "last mile" connections to ports and airports.

RFI (Rete Ferroviaria Italiana - Italian Railway Network) has been undertaking for several years an intense activity of maintenance and renewal of the infrastructure heritage. On the other hand, ETS is an engineering company with a strong background started in the railway industry.

RFI has foreseen a difficult, long and challenging work plan for the Italian infrastructure. In order to guarantee efficient investments, the mission of ETS has been to support the renewal of the strategic railway lines. In this context, ARCHITA was born. It was designed to satisfy the customer needs to reduce the time of knowledge of the infrastructural heritage and for its decision-making, focusing only on the most critical and strategic railway lines (on a small scale). ARCHITA has given fast and reliable outputs to support design solutions and works strategy (large scale) with preliminary economic evaluation through infrastructure and structures detection, maintenance estimation, slope stability assessment and clearance analyses.

\footnotetext{
* Corresponding author: federico.foria@etsingegneria.it
} 


\section{ARCHITA}

\subsection{Devices and configurations}

ARCHITA is a multi-dimensional mobile mapping system developed by ETS consisting of linked and integrated equipment:

- Survey sensors: laser scanner, high-resolution photo, thermal image cameras, georadars; - Positioning sensors: GPS, IMU, Odometer (they are integrated and work with a "tightly coupled" Kalman filter that accepts additional information as Ground Control Points (GCP) in post-processing. In this way, the trajectories are corrected through adjustment procedures.

ARCHITA uses the GNSS+IMU sensors to calculate the moving trajectory in a predefined reference system. The position and attitude of a particular point of the system, through the knowledge of appropriate lever-arms, is recalculated with all the related acquisitions (i.e. photographic images, thermal images and cloud points).

The precision of the system depends on the positioning precision of the GNSS system. This one depends on various factors, such as: the number, type, visibility and layout of the satellites, and the eventual absence of GPS signal (outage). In ideal conditions (open sky) and without interruption of the signal, the positioning precision of the trajectory can be equal to $2 \mathrm{~cm}$.

ARCHITA carries out the survey activity with an average speed of $15-30 \mathrm{~km} / \mathrm{h}$, minimizing the impact on existing lines with shortstop of the traffic and without disconnection of the electrical tension (for railway). The system can work in two different configurations.

RAIL configuration is equipped with:

- Laser Scanner (Leica Pegasus:Two), in configuration with n. 8 digital cameras, 1 IMU inertial platform, 2 profiler Z + F 9012, 2 GPS antennas, 1 optical odometer and 4 thermal imaging cameras. The two profilers, arranged at $30^{\circ}$ and $60^{\circ}$ to the axis of the binary track, allow a reduction of the "shadows".

- Ground Penetrating Radar (SRS SafeRail System by I.D.S. GeoRadar), in the configuration of n.3 radar antennas characterized by $400 \mathrm{MHz}$ central frequency.

- Ground Penetrating Radar (by I.D.S. GeoRadar), in the configuration of n.1 radar antenna characterized by $600 \mathrm{MHz}$ central frequency.

- HD images (by ADTS), in configuration of 3 high-resolution linear cameras, 1 profiler Z + F 9012 and lighting system composed of n.16 LED light, on steel structure and aligned with the cameras.

ROAD configuration is equipped with:

- Laser Scanner (Leica Pegasus:Two), in configuration with n. 8 digital cameras, 1 IMU inertial platform, 1 profiler Z + F 9012, 1 GPS antennas, 1 odometer.

- Ground Penetrating Radar (Hi Pave System by I.D.S. GeoRadar), in the configuration of $\mathrm{n} .1$ radar antenna characterized by $2 \mathrm{GHz}$ central frequency and $\mathrm{n} .1$ antenna characterized by $400 / 900 \mathrm{MHz}$ central frequency.

- Ground Penetrating Radar (by I.D.S. GeoRadar), in the configuration of n.1 radar antenna characterized by $600 \mathrm{MHz}$ central frequency.

- HD images (by ADTS), in configuration of 3 high-resolution linear cameras, 1 profiler Z + F 9012 and lighting system composed of n.16 LED light, on steel structure and aligned with the cameras. 

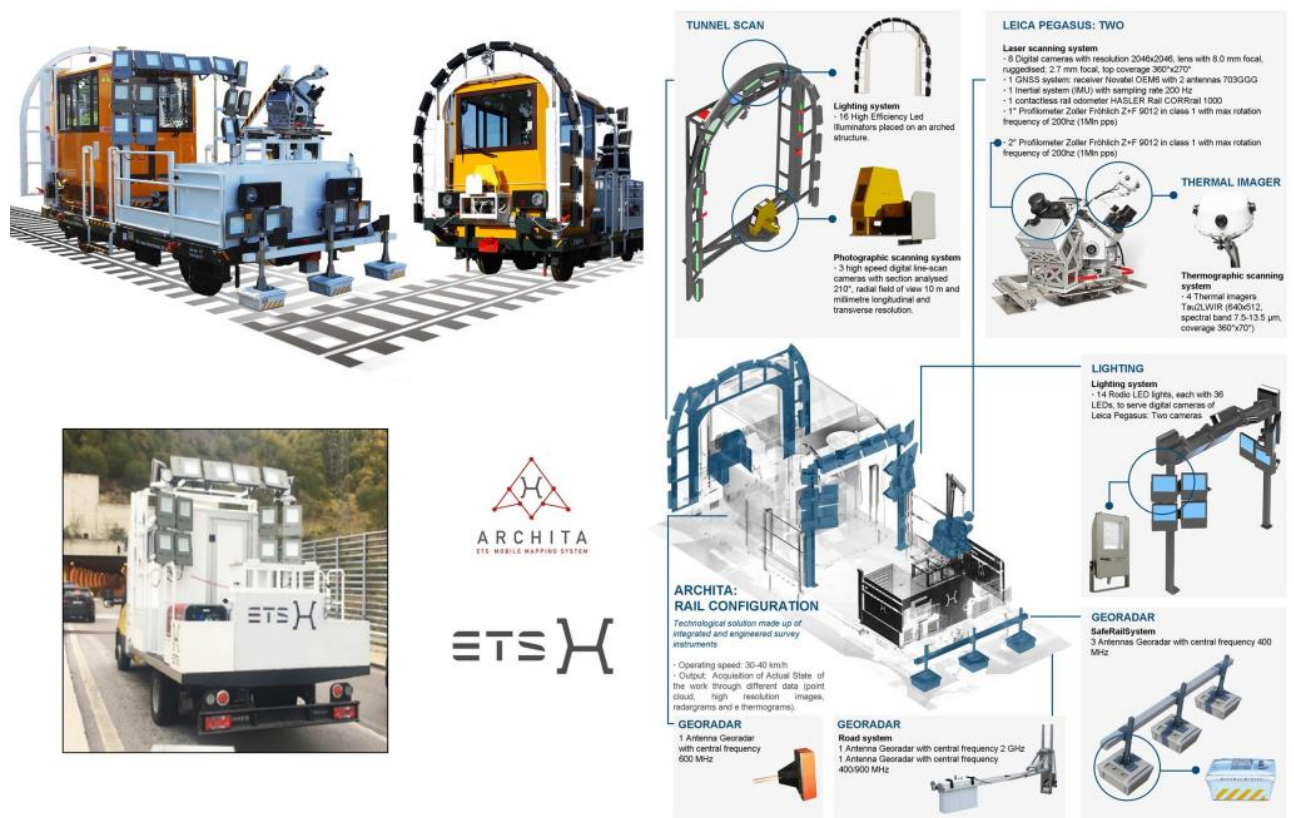

Fig. 1. ARCHITA equipment (RAIL/ROAD configuration).

ARCHITA can obtain a series of integrated and connected information in a single passage, such as:

- Geometry (Figure 2): georeferenced 3D point cloud. In post-processing, it's possible to obtain the track geometry and the correspondent CAD models (i.e. tunnel shape, clearance analysis, track, electric supports and contact line).
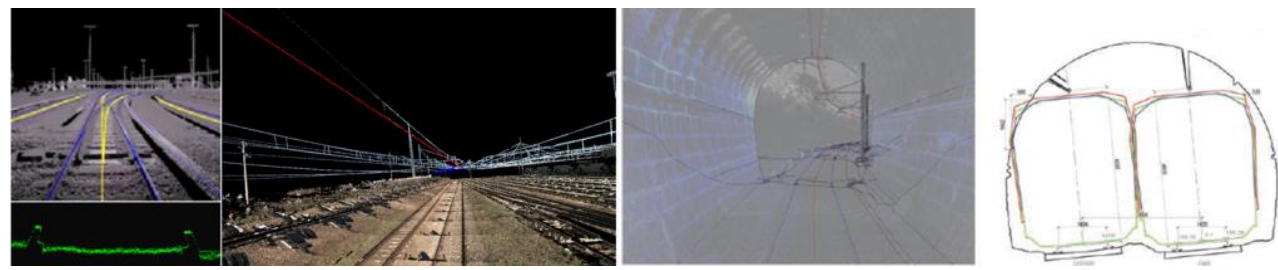

Fig. 2. Galleria Sipicciano and Galleria Olmata (Italy): outputs from 3D point cloud.

- Ballast (RAIL configuration, Figure 3): 400-600 MHz georadar, for the definition of the thickness, material alterations, identification of cavities, and presence of water.

- Lining (Figure 4): $600 \mathrm{MHz}$ georadar, for the definition of the thickness, identification of cavities, material alterations and presence of water.

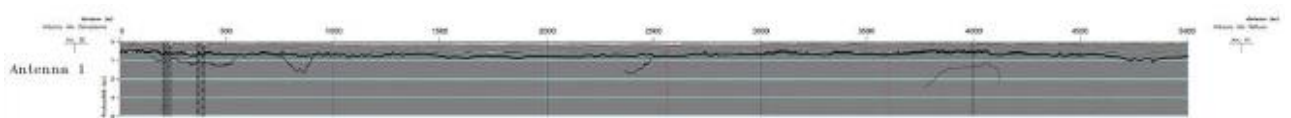

Fig. 3. Roma-Cassino railway line (Italy): GPR survey on the ballast.

Fig. 4. Galleria Sipicciano (Italy): GPR processing survey on the tunnel lining. 
- Lining condition: high-definition photo (Figure 5) and thermographic analysis (Figure 6) synchronized with the $3 \mathrm{D}$ point cloud.

- Pavement (ROAD configuration): $2.0 \mathrm{GHz}$ georadar, suitable frequency for the definition of the asphalt thickness and 400/900 MHz georadar, suitable frequency for the definition of the subgrade thickness.

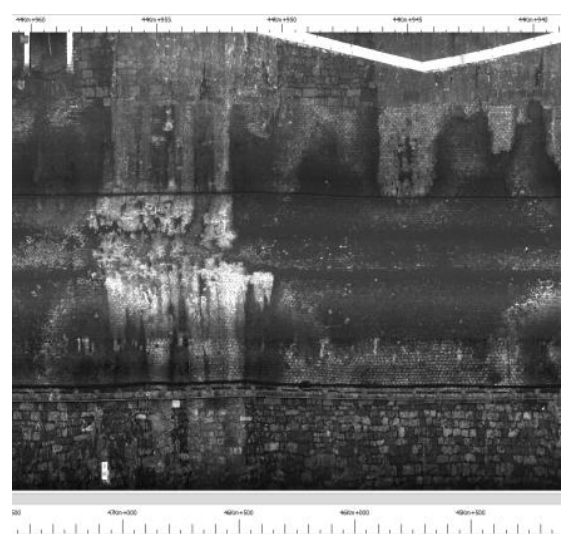

Fig. 5. Galleria Montorso (Italy): HD photographich images.

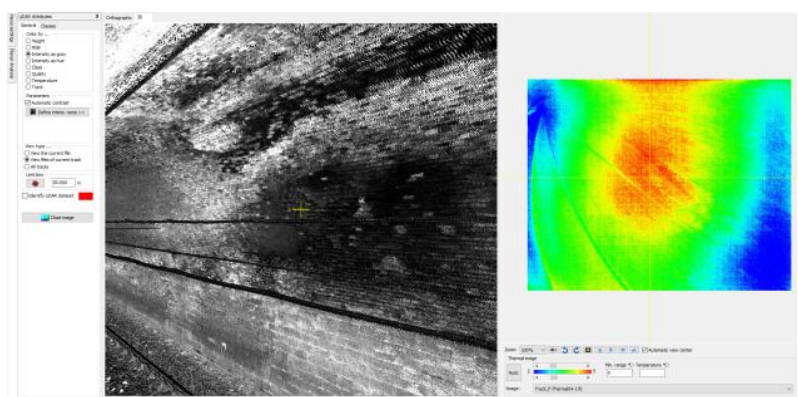

Fig. 6. Galleria Montorso (Italy): 3D point cloud synchronized with thermographic images.

\subsection{Product and software development}

ARCHITA has passed through a severe process of development (still on-going). Leica Geosystems customized the laser scanner adding n.1 profiler, n.1 GPS antenna and n.4 thermal cameras. ADTS customized the Tunnel Scan adding n.1 profiler and n.1 GPS antenna.

Bruno Kessler Foundation (FBK) coordinated the integration between the Laser Scanner (Leica Geosystems) and the Tunnel Scan (ADTS). Bruno Kessler Foundation (FBK) has demonstrated successfully the integration through a field campaign on the railway line Attigliano-Viterbo (the integration tests were performed in the Sipicciano I and Sipicciano II tunnels in Italy). This study proved that the 3D point clouds of the two instruments have a maximum deviation of $1.5 \mathrm{~cm}$.

The development has also covered the elaboration software: Leica Geosystems customized the post-processing software based on the new configuration of the product. ADTS has been customizing the post-processing software based on the specific requests of ETS and the clients.

The style for the names is First Names, typed in italic 10-point Times, then Last Name, typed in 10-point Times, with a comma after all except the last author, which is separated by comma + "and". Do not use academic titles. 


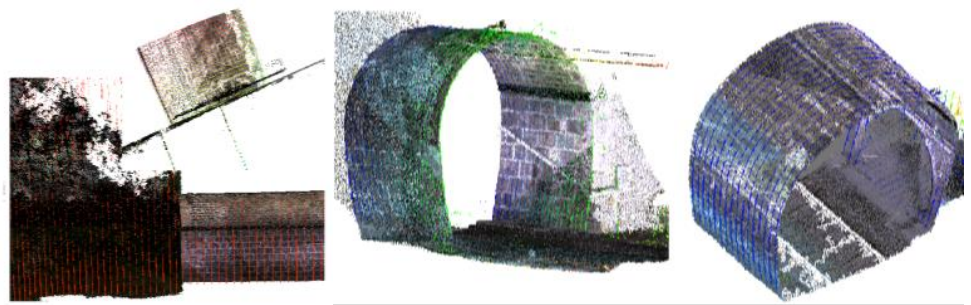

Fig. 7. Galleria Sipicciano (Italy): results of point cloud integration (Tunnel Scan - Pegasus:Two).

\section{Application: geometric survey and clearance analysis for DTP Roma, Italy}

\subsection{Problem and requirements}

ETS has been in charge to investigate for the DTP Roma (RFI Lazio railway district), which railway line between Rome and Naples is more convenient to renew. This feasibility study and the related project are part of the Scandinavian-Mediterranean Corridor, which represents the north-south axis of the Trans-European Transport Networks (TEN-T).

The project required: the geometric survey of the railway infrastructure, the widening of the clearance to ensure the strengthening of the railway, the resolution of the leakages, the mapping and backfilling of the cavities along the line and the execution of the works with respect to a working line. ETS has carried out a geometric and knowledge detection of about $1200 \mathrm{~km}$ of the railway line (built in the early 1900s) of which $170 \mathrm{~km}$ of tunnels (mainly with masonry lining), overhead lines and railway stations.

\subsection{Survey of geometries and lining conditions}

ARCHITA has been used to determine the inner geometry (Figure 8) in order to support the check of the clearance conditions to design the milling of the existing lining and, consequently, the appropriate reinforcement works.
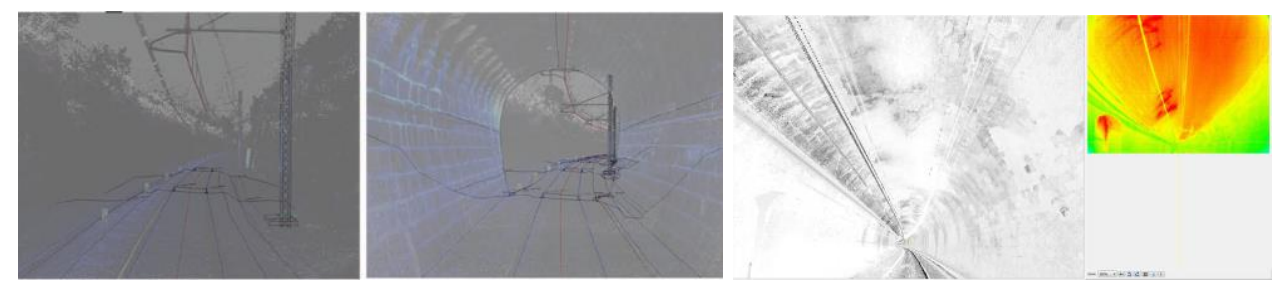

Fig. 8. Galleria Sipicciano (Italy): geometry reconstruction from ARCHITA point cloud to 3D digitalization and thermographic result.

In Figure 9, it's depicted the output of high-resolution photo to check the condition and the humidity content of the lining. In the same figure, it's shown a georadar elaboration to determine the thickness of the lining. The thicknesses have been verified and calibrated based on traditional destructive tests.

The ARCHITA outputs have been integrated in a BIM approach through all the stages of survey and design (Figure 10). ARCHITA has been used to check both ballast and lining thicknesses and condition

All these outputs support the decision-maker towards the more convenient line to renew and the designers towards the more appropriate interventions. 

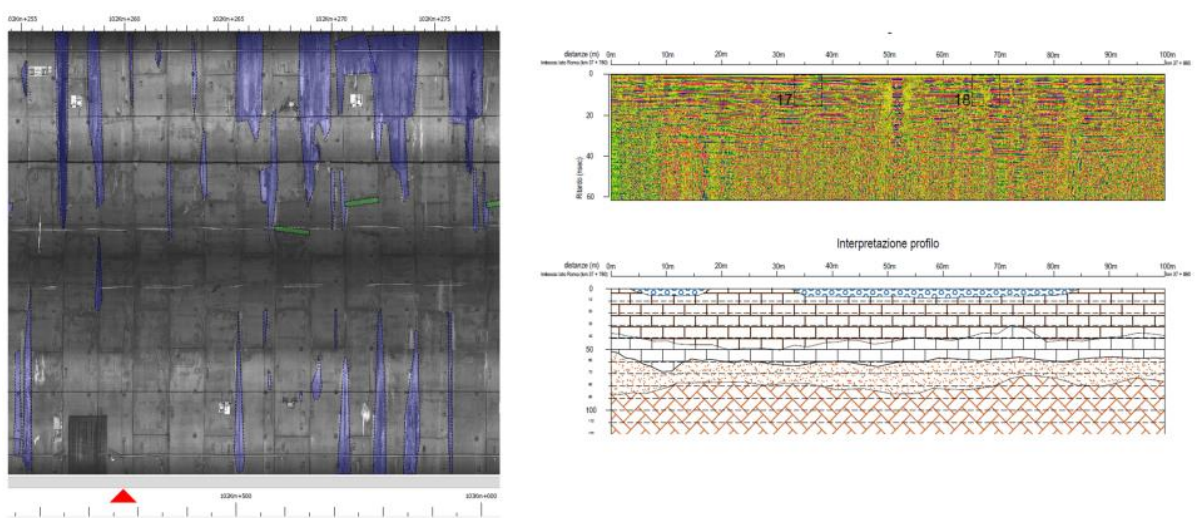

Fig. 9. Galleria Costa dei Rosi (Italy): high-resolution photo for the defects detection (left) and GPR for lining thickness (right).

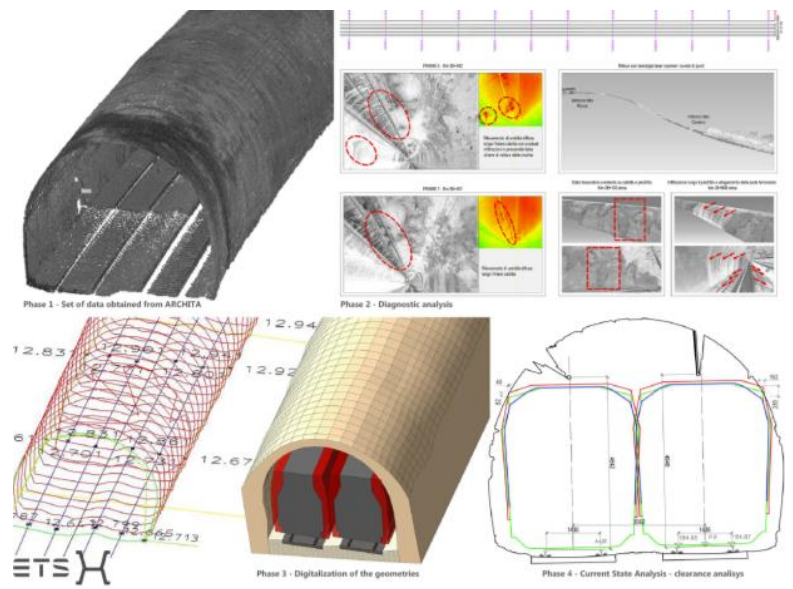

Fig. 10. Galleria Olmata (Italy): workflow for "BIM oriented" approach.

\section{Conclusions}

The underground space has already been widely used in the past centuries. The number of existing tunnels requiring renewal, monitoring or maintenance, increases day by day. For this reason, ETS has been developing ARCHITA. The system guarantees a quick and reliable integrated survey of the existing infrastructure to help design and feasibility studies.

Actually, ARCHITA has been deployed in more than $400 \mathrm{~km}$ of tunnels and $2000 \mathrm{~km}$ of infrastructure in Italy. One case has been briefly presented. Nevertheless, ARCHITA is proving that still a lot needs to be done to achieve a clear, objective and repeatable way to survey and manage the existing tunnels and infrastructure.

\section{References}

1. M. Gavilán, F. Sánchez, J.A. Ramosand, O. Marcos, The 6th International Conference on Structural Health Monitoring of Intelligent Infrastructure (2013)

2. G. Loprencipe, L. Moretti, T. Pestillo, R. Ferraro, Sustainability, E 10, 3145 (2018)

3. R. Montero, J.G. Victores, S. Martínez, A. Jardón, C. Balaguer, Automation in Construction, E 59, 99-112 (2015) 